\title{
Erbium implanted silicon rich oxide thin films suitable for slot waveguides applications
}

\author{
Nikola Prtljaga a,*, Daniel Navarro-Urrios ${ }^{\mathrm{b}}$, Alessandro Marconi ${ }^{\mathrm{a}}$, Aleksei Anopchenko ${ }^{\mathrm{a}}$, \\ Jean-Philippe Colonna ${ }^{c}$, Frédéric Milesi ${ }^{c}$, Nicola Daldosso ${ }^{a}$, Olivier Jambois ${ }^{b}$, Blas Garrido ${ }^{b}$, \\ Jean-Marc Fedeli ${ }^{\mathrm{c}}$, Lorenzo Pavesi ${ }^{\mathrm{a}}$ \\ ${ }^{a}$ Nanoscienze Laboratory, Department of Physics, University of Trento, Via Sommarive 14, Povo (Trento) 38 121, Italy \\ ${ }^{\mathrm{b}}$ MIND-IN2UB, Departament d'Electrònica, Universitat de Barcelona, Carrer Martì i Franquès 1, Barcelona 08028, Spain \\ ${ }^{\text {c } C E A, ~ L E T I, ~ M i n a t e c ~-~} 17$ Rue des Martyrs, 38054 Grenoble Cedex 9, France
}

\section{A R T I C L E I N F O}

\section{Article history:}

Received 4 June 2010

Received in revised form 15 November 2010

Accepted 16 December 2010

Available online 17 January 2011

Keywords:

Si nanocrystals

Erbium

Thin film

Slot waveguide

\begin{abstract}
A B S T R A C T
Thin $(50 \mathrm{~nm})$ erbium implanted silicon rich oxide films suitable for slot waveguides applications have been produced and studied by means of optical spectroscopy and structural characterisation techniques. Comparison between different deposition techniques in terms of light emitting properties of erbium ions is presented. Special attention is given to the efficiency improvement of the energy transfer from silicon nanoclusters to erbium ions where the type of annealing treatment is proven to be of crucial importance.
\end{abstract} (C) 2010 Elsevier B.V. All rights reserved.

\section{Introduction}

Silicon photonics has experienced a rapid development in the last decade imposing itself as a future key technology in the "beyond CMOS" domain and natural point of convergence for a CMOS based nanoelectronics [1]. Despite the fact that the demonstration of the basic single building blocks has been approaching to its closure and integration of the optical components on CMOS platform is on the way, a monolithically integrated and efficient all silicon light source is still lacking [2].

Although, Si-nc have been recognised as a possible gain medium [3], their spectral emission region is not suited for direct signalling within optical fibers, i.e. for telecommunication applications. However, co-doping with erbium ions has been proven as a viable path towards a spectral tailoring in the more desirable $1.55 \mu \mathrm{m}$ spectral region (third telecom window) [4]. The potentiality of this approach has been largely restricted due to its inherent need for optical pumping. In fact, Si-nc are formed in an oxide matrix through which current does not flow easily. Thick layers are requested for light confinement in waveguide structures [5] while thin layers are needed for electrical injection [6]. The situation changed when slot waveguides were demonstrated offering

\footnotetext{
* Corresponding author. Tel.: +39 0461283957; fax: +39 0461282957.

E-mail address: nikolap@science.unitn.it (N. Prtljaga).
}

the possibility of electrical injection and increased light confinement in the gain region [7]. Slot waveguides are formed by two thick silicon layers separated by a thin erbium doped silicon rich oxide (SRO) layer where most of the optical mode is confined. From the initial theoretical proposal [8], little has been done to assess the suitability of the optimal thick film parameters to such configuration [5].

The aim of this work is to fill this gap by reporting the results of a characterisation of thin $(\sim 50 \mathrm{~nm})$ erbium implanted SRO films. Comparison between different deposition techniques and evaluation of optimum thermal treatment is presented.

\section{Experimental}

The samples were prepared by using three different deposition techniques: PECVD (Plasma Enhanced Chemical Vapour Deposition), LPCVD (Low Pressure Chemical Vapour Deposition) and silicon ion implantation in stoichiometric silicon oxide produced by LPCVD. All the thin films were deposited on p-type CZ silicon substrate $(1-50 \Omega \mathrm{cm})$ while deposition parameters were adjusted in order to produce different Si excess in the SRO (see Table 1).

After the deposition, all samples were thermally treated to yield phase separation, silicon precipitation and nanocluster formation. The type, temperature and duration of the thermal treatments were varied. In the erbium co-doped samples, erbium was 
Table 1

Parameters of characterized samples.

\begin{tabular}{|c|c|c|c|c|}
\hline Name & Deposition technique & Nominal Si excess (at.\%) & Annealing: type/temperature/duration & Post-annealing: type/temperature/duration \\
\hline Ref. [1] & LPCVD & 0 & $\mathrm{RTP} / 900^{\circ} \mathrm{C} / 5^{\prime}$ & $\mathrm{RTP} / 1100^{\circ} \mathrm{C} / 5^{\prime}$ \\
\hline Ref. [2] & - & 100 & $\mathrm{RTP} / 900{ }^{\circ} \mathrm{C} / 5^{\prime}$ & $\mathrm{RTP} / 1100^{\circ} \mathrm{C} / 5^{\prime}$ \\
\hline Ref. [3] & PECVD & 16 & $\mathrm{RTP} / 900{ }^{\circ} \mathrm{C} / 5^{\prime}$ & $\mathrm{RTP} / 1100^{\circ} \mathrm{C} / 5^{\prime}$ \\
\hline A & LPCVD & 12 & $\mathrm{RTP} / 1100^{\circ} \mathrm{C} / 5^{\prime}$ & Furnace $/ 800^{\circ} \mathrm{C} / 6 \mathrm{~h}$ \\
\hline B & Si I.I. & 12 & $\mathrm{RTP} / 1100^{\circ} \mathrm{C} / 5^{\prime}$ & Furnace $/ 800^{\circ} \mathrm{C} / 6 \mathrm{~h}$ \\
\hline $\mathrm{C}$ & PECVD & 12 & $\mathrm{RTP} / 1100^{\circ} \mathrm{C} / 5^{\prime}$ & Furnace $/ 800^{\circ} \mathrm{C} / 6 \mathrm{~h}$ \\
\hline $\mathrm{A} 1$ & LPCVD & 12 & Furnace $/ 900^{\circ} \mathrm{C} / 1 \mathrm{~h}$ & Furnace $/ 800^{\circ} \mathrm{C} / 6 \mathrm{~h}$ \\
\hline $\mathrm{C} 1$ & PECVD & 12 & Furnace $/ 900^{\circ} \mathrm{C} / 1 \mathrm{~h}$ & Furnace $/ 800^{\circ} \mathrm{C} / 6 \mathrm{~h}$ \\
\hline A2 & LPCVD & 12 & Furnace $/ 900^{\circ} \mathrm{C} / 1 \mathrm{~h}$ & Furnace $/ 800^{\circ} \mathrm{C} / 6 \mathrm{~h}$ \\
\hline $\mathrm{C} 2$ & PECVD & 12 & Furnace $/ 900^{\circ} \mathrm{C} / 1 \mathrm{~h}$ & Furnace $/ 800^{\circ} \mathrm{C} / 6 \mathrm{~h}$ \\
\hline
\end{tabular}

introduced using ion implantation (Dose: $1 \times 10^{15} \mathrm{at} . / \mathrm{cm}^{2}$, Energy: $40 \mathrm{keV}$ (reference samples) or $25 \mathrm{keV}$ (other samples)). After implantation, samples were additionally annealed to recover implantation induced defects and to activate implanted erbium ions. The temperature of post-annealing process was kept constant among the different samples. Three reference samples were produced (Refs. [1-3], see Table 1).

Recently, for thick samples it has been reported that light emitting properties of erbium based material could significantly change inside slot waveguide [9] or close to a plane of high refractive index material [10]. This might cause discrepancies between optimisation of thin films when they are inserted into slot waveguide. Thus to avoid this problem we covered the samples by a $150 \mathrm{~nm}$ thick polysilicon layer in order to provide a "slot like" dielectric environment.

The slot thickness of $50 \mathrm{~nm}$ has been chosen as a trade-off between the maximum value for which efficient bipolar injection has been demonstrated [6] and the optimum value for the minimum laser threshold [11].

The determination of the percentage of the silicon excess was done by means of XPS measurements, whereas SIMS was employed for determination of erbium concentration. The silicon excess was calculated with the formula $(1-x / 2) /(1+x)$, with $x=[0] /[\mathrm{Si}]$ as measured by XPS. Erbium concentrations were determined by using a calibration sample of known erbium concentration.

The continuous photoluminescence (CW PL) measurements were performed using the lock-in technique, with the $476 \mathrm{~nm}$ line of an Argon ion laser (Coherent I90C-4) which is not resonant with any erbium optical transition. Time resolved photoluminescence (TR PL) measurements were done by modulating a laser beam with a mechanical chopper on higher frequency. The detection system consisted of: spectrograph coupled to InGaAs PMT and photon counting unit. CW and TR PL measurements were performed at the same photon fluxes $\left(4 \times 10^{20}\right.$ photons $\left./ \mathrm{cm}^{2}\right)$.

\section{Results}

In order to evaluate the suitability of different deposition technique in producing high quality thin films, three nominally equal samples were produced by different techniques and analyzed (samples A, B and C in Table 1 and Fig. 1). All of three samples were implanted by the same erbium dose and thermally treated in the same way (RTP annealing). Lower Si excess was chosen (12 at.\%). The silicon excess was determined by XPS measurements on monitor samples (data not shown). In the case of LPCVD and PECVD, actual silicon excess can deviate up to few percent from nominal value of 16 at.\%.

Although, the exact knowledge of the size, density and fraction of clustered silicon is certainly of great help in order to study the energy transfer efficiency, we employ a more phenomenological approach. It has been shown [12] that the size, density and percentage of clustered silicon in samples produced by different

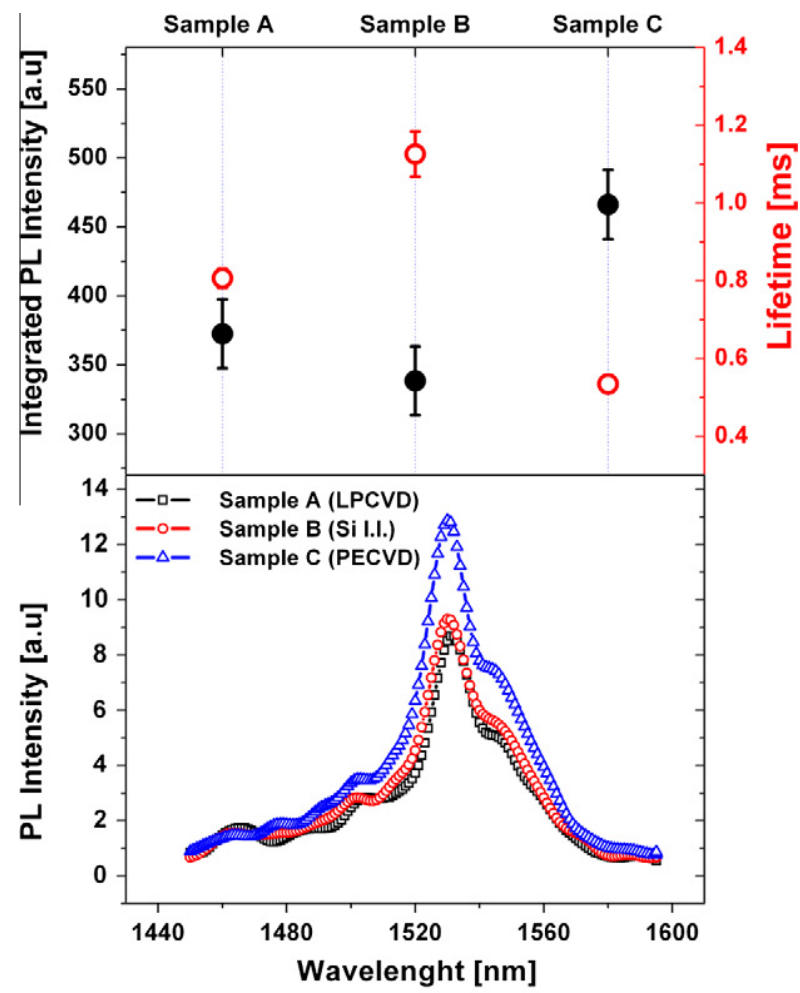

Fig. 1. (Top) Integrated IR PL intensity (full black circles) and corresponding decay constants (empty red circles) for the samples A, B and C. (Bottom) PL spectra of sample A (empty black squares), sample B (empty red circles) and sample C (empty blue triangles). Excitation wavelength was $476 \mathrm{~nm}$ in all cases. (For interpretation of the references to colour in this figure legend, the reader is referred to the web version of this article.)

deposition techniques influence strongly the light emitting properties of Si nanoclusters. Therefore, we compare samples with different local environment of erbium ions by monitoring structural parameters (silicon excess, erbium concentration profile) and visible PL (not reported here). Then we monitor the erbium ions emission excited by non-resonant excitation, i.e. via energy transfer from the Si-nc.

When exciting with $476 \mathrm{~nm}$ light the two reference samples (erbium in $\mathrm{SiO}_{2}$ Ref. [1] and erbium in silicon substrate Ref. [2]) show no PL signal, assuring that the photoluminescence signal observed in other samples (see Table 1) is coming from erbium ions excited indirectly by an energy transfer from the silicon nanoclusters. Typical PL spectra and decay constants are shown in Fig. 1. Decay constants are of the order of millisecond, somewhat shorter than what is usually reported for thick samples [5].

The best sample in terms of PL intensity is the one deposited by PECVD. The same sample also shows the fastest decay and highest sensibility to a change in annealing conditions (Fig. 2). This could 


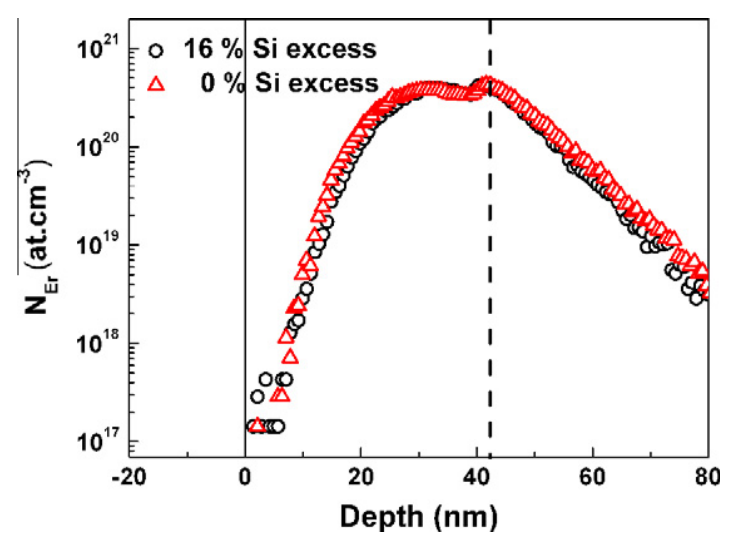

Fig. 2. Erbium concentration profiles in Ref. [3] (empty black circles) and Ref. [2] sample (empty red triangles). Vertical solid black line corresponds to an air/sample interface while the vertical dashed black line indicates thin film/silicon substrate surface. (For interpretation of the references to colour in this figure legend, the reader is referred to the web version of this article.)

be an indication of a low matrix quality due to incorporation of nitrogen during the deposition process.

From XPS data obtained on monitor samples we found that the presence of nitrogen in our PECVD samples could be as high as 56 at.\% while not detectable in the samples deposited by the other two techniques. The presence of nitrogen could offset the silicon mobility thus requiring higher annealing temperatures to form the Si-nc and slowing down the matrix restructuring [13].

Most of the difference in the PL intensity between these three samples could be simply attributed to a difference in the decay constants.

The longest decay times is observed for sample deposited by $\mathrm{Si}$ ion implantation. Although, this sample was double implanted (first by silicon ions and subsequently by erbium ones) the postannealing treatments applied was sufficient for complete matrix recovery.

Since for electrical injection, multilayer $\mathrm{Si}-\mathrm{nc} / \mathrm{SiO}_{2}$ films have better properties in terms of low turn-on voltage of electroluminescence $[6,14]$ and since silicon ion implantation is not suitable for multilayer sample production, we focused additional attention to PECVD and LPCVD samples. In particular, another type of annealing (furnace annealing) was studied while varying the Si excess in the samples.

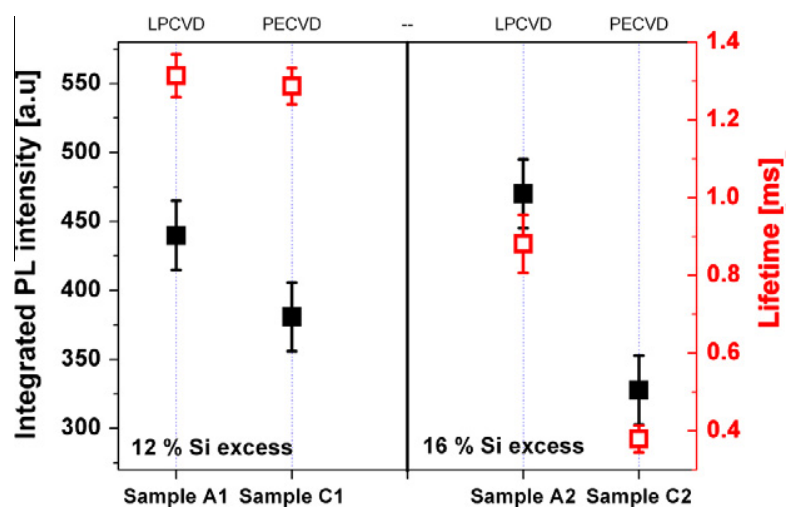

Fig. 3. Integrated IR PL intensity (full black squares) and corresponding decay constants (empty red squares) for samples A1, C1(left) and samples A2, C2 (right). Excitation wavelength was $476 \mathrm{~nm}$ in all cases while the other experimental conditions are the same as for Fig. 1, so the data are mutually comparable. (For interpretation of the references to colour in this figure legend, the reader is referred to the web version of this article.)
As the influence of different silicon excess on erbium ion distribution in thin films is not known, two reference samples (Refs. $[1,3]$, see Table 1 ) were produced to study this effect. As can be seen in Fig. 2, erbium concentration distribution is not influenced by the silicon excess, not even at high percentage. This result suggests that the difference in light emitting properties among the different samples is mainly due to a different silicon clusterization and erbium ions - Si-nc interaction.

The furnace annealing at $900{ }^{\circ} \mathrm{C}$ for $1 \mathrm{~h}$ is the most suitable annealing for $500 \mathrm{~nm}$ thick samples [5]. In the case of thin samples with 12 at.\% of silicon excess, furnace annealing at $900{ }^{\circ} \mathrm{C}$ seems to be beneficial with respect to RTP annealing especially in the case of the LPCVD sample (A1) (Fig. 3). Both, A1 and C1 sample show similar PL intensities while decay constant reached the level of the sample B indicating much better matrix quality. With the same annealing and increased silicon excess, degradation in light emitting properties is observed. The decay constants diminish in both samples (A2 and C2), while in sample C2 PL intensity decreases as well.

\section{Conclusions}

Based on the results presented, oxide matrix quality is of key importance. The decay constants in our samples indicate good oxide quality and weak non-radiative recombinations. Best results are found for samples where erbium ions are in impurities free (nitrogen-free) stoichiometric $\mathrm{SiO}_{2}$, which is the case of silicon ion implantation and LPCVD; LPCVD being more suited for multilayer approach. These results agree well with what was recently found on similar materials [15]. While erbium concentration distribution is independent on silicon excess, higher silicon cluster densities impact negatively on light emitting properties of erbium ions. The same decrease in performances is observed when a partial phase separation is present. For this reason, furnace annealing looks more appropriate with respect to rapid thermal annealing to get a complete phase separation.

\section{Acknowledgments}

This work is supported by HELIOS Project (FP7 224312) and IT09ILB2F9 (Integrated Italia - Spain action) Project.

\section{References}

[1] Consultation Report of FP7 Workshop on Advanced Nanoelectronics Technologies, European Commission, Brussels, 11 September, 2009.

[2] R. Soref, Advances in Optical Technologies, Article ID 472305, 2008, pp. 7

[3] L. Pavesi, L. Dal Negro, C. Mazzoleni, G. Franzò, F. Priolo, Nature 408 (2000) $440-443$.

[4] J. Lee, J. Shin, N. Park, J. Lightwave Technol. 23 (1) (2005) 19-25.

[5] N. Daldosso, D. Navarro-Urrios, M. Melchiorri, C. Garcìa, P. Pellegrino, B. Garrido, C. Sada, G. Battaglin, F. Gourbilleau, R. Rizk, L. Pavesi, IEEE J. Sel. Top. Quant. 12 (6) (2006) 1607-1617.

[6] A. Anopchenko, A. Marconi, E. Moser, S. Prezioso, M. Wang, L. Pavesi, G. Pucker, P. Bellutti, J. Appl. Phys. 106 (2009) 033104.

[7] Q. Xu, V.R. Almeida, R.R. Panepucci, M. Lipson, Opt. Lett. 29 (15) (2004) 16261628.

[8] C.A. Barrios, M. Lipson, Opt. Exp. 13 (25) (2005) 10092-10101.

[9] Y.C. Jun, R.M. Briggs, H.A. Atwater, M.L. Brongersma, Opt. Exp. 17 (9) (2009) 7479-7490.

[10] P. Horak, W.H. Loh, A.J. Kenyon, Opt. Exp. 17 (2) (2009) 906-911.

[11] J.T. Robinson, K. Preston, O. Painter, M. Lipson, Opt. Exp. 16 (21) (2008) 1665916669.

[12] G. Franzò, M. Miritello, S. Boninelli, R. Lo Savio, M.G. Grimaldi, F. Priolo, F. Iacona, G. Nicotra, C. Spinella, S. Coffa, J. Appl. Phys. 104 (2008) 094306.

[13] N. Daldosso, G. Das, S. Larcheri, G. Mariotto, G. Dalba, L. Pavesi, A. Irrera, F. Priolo, F. Iacona, F. Rocca, J. Appl. Phys. 101 (2007) 113510.

[14] M. Wang, A. Anopchenko, A. Marconi, E. Moser, S. Prezioso, L. Pavesi, G. Pucker, P. Bellutti, L. Vanzetti, Phys. E 41 (2009) 912-915.

[15] A. Irrera, F. Iacona, G. Franzò, M. Miritello, R. Lo Savio, M.E. Castagna, S. Coffa, F. Priolo, J. Appl. Phys. 107 (2010) 054302. 